\title{
Equilibrium Asset and Option Pricing under Jump-Diffusion Model with Stochastic Volatility
}

\author{
Xinfeng Ruan, ${ }^{1}$ Wenli Zhu, ${ }^{1}$ Shuang $\mathrm{Li}^{2}{ }^{2}$ and Jiexiang Huang ${ }^{1}$ \\ ${ }^{1}$ School of Economic Mathematics, Southwestern University of Finance and Economics, Chengdu 611130, China \\ ${ }^{2}$ Department of Mathematics \& Statistics, Curtin University, Perth, WA 6102, Australia \\ Correspondence should be addressed to Xinfeng Ruan; ruanxinf@gmail.com
}

Received 24 July 2013; Revised 30 September 2013; Accepted 10 October 2013

Academic Editor: Yong Hong Wu

Copyright (c) 2013 Xinfeng Ruan et al. This is an open access article distributed under the Creative Commons Attribution License, which permits unrestricted use, distribution, and reproduction in any medium, provided the original work is properly cited.

\begin{abstract}
We study the equity premium and option pricing under jump-diffusion model with stochastic volatility based on the model in Zhang et al. 2012. We obtain the pricing kernel which acts like the physical and risk-neutral densities and the moments in the economy. Moreover, the exact expression of option valuation is derived by the Fourier transformation method. We also discuss the relationship of central moments between the physical measure and the risk-neutral measure. Our numerical results show that our model is more realistic than the previous model.
\end{abstract}

\section{Introduction}

Option pricing problem is one of the predominant concerns in the financial market. Since the advent of the Black-Scholes option pricing formula in [1], there has been an increasing amount of literature describing the theory and its practice. Due to drawbacks of the Black-Scholes model which cannot explain numerous empirical facts such as large and sudden movements in prices, heavy tails, volatility clustering, the incompleteness of markets, and the concentration of losses in a few large downward moves, many option valuation models have been proposed and tested to fit those empirical facts. Jump-diffusion models with stochastic volatility could overcome these drawbacks of the Black-Scholes model in [221]. Based on those advantages, in this paper, we focus on studying the jump-diffusion model with stochastic volatility.

Different from the Black-Scholes framework, we use jump diffusion to describe the price dynamics of underlying asset. The market of our model is incomplete; that is, it is not possible to replicate the payoff of every contingent claim by a portfolio, and there are several equivalent martingale measures. How to choose a consistent pricing measure from the set of equivalent martingale measures becomes an important problem. This means that we need to find some criteria to determine one from the set of equivalent martingale measures in some economically or mathematically motivated fashion. A unique martingale measure was found by various researchers via using optimal criteria, for instance, minimal martingale criterion, minimal entropy martingale criterion, and utility maximization criterion [22-31].

General equilibrium framework method is also a popular method to deal with the option pricing in an incomplete market. General equilibrium framework is initially introduced by Lucas Jr. (1978) [32], Cox et al. (1985) [33] and developed by Vasanttilak and Lee (1990) [34], Pan (2002) [35], Liu and Pan (2003) [36], Liu et al. (2005) [37], Bates (2008) [38], SantaClara and Yan (2004) [12], and Zhang et al. (2012) [6]. They assumed that there is a representative investor who wants to maximize an objective function in a rational expectations economy where there are one risk-free asset and one risky asset. When the market is clear, the representative investor takes all money into the risky asset. In this paper, we build a general equilibrium model which is the same as that due to Santa-Clara and Yan (2004) [12]. Under this model, we obtain an exact expression of the equity premium and the pricing kernel in a general equilibrium economy. This can be regarded as a great contribution to the literature.

The pricing kernel which acts like the physical and riskneutral densities and moments in the economy is also a vitally important problem in mathematical finance. In some 
constant volatility models with jump diffusions, Pan (2002), Liu and Pan (2003), and Liu et al. (2005) [35-37] derived the pricing kernel with some restrictions of jump sizes in a general equilibrium setting. Recently, Zhang et al. (2012) [6] presented an analytical form for the pricing kernel without any distributional assumption on the jumps. In this paper, we extend the results of Zhang et al. (2012) to the pricing kernel with stochastic volatility.

Duffie et al. (2000) [10] and Chacko and Das (2002) [39] presented a transform analysis to price the valuation of options for affine jump diffusions with stochastic volatility. Lorig and Lozano-Carbasse (2013) [21] studied option pricing in exponential Lévy-type models with stochastic volatility and stochastic jump intensity. Lewis (2008) [8] used Fourier transformation methods to obtain the transformbased solution of option price. In this paper, we employ the Fourier transformation method to get the exact expression of European options.

Finally, we get the relationship of central moments between the physical measure and the risk-neutral measure which can help us to study the negative variance risk premium, the implied volatility smirk, and the prediction of realized skewness. Some relevant work has been done by Bakshi et al. (2003) [13], Carr and Wu (2009) [14], and Neuberger (2012) [15]. However, to the best of our knowledge, except for Zhang et al. (2012) [6], there is no literature studying this relationship. In this paper, we extend it to a stochastic volatility case. This can be regarded as another contribution to the literature.

The rest of the paper is organized as follows. In Section 2, we present our jump-diffusion model with stochastic volatility. In Section 3, we study the equity premium in a general equilibrium economy. The option pricing and the relationship of central moments between the physical measure and the risk-neutral measure are studied in Section 4. Numerical results and conclusions are shown in Sections 5 and 6, respectively.

\section{Our Model}

In this paper, we consider the financial market with the following two basic assets.

(i) $A$ Bond whose price $P_{t}$ at time $t$ is given by

$$
d P_{t}=r P_{t} d t, \quad P_{0}=1 .
$$

(ii) A Stock whose price $S_{t}$ at time $t$ is given by

$$
\begin{gathered}
\frac{d S_{t}}{S_{t}}=\mu d t+\sqrt{V_{t}} d B_{t}{ }^{S}+\left(e^{x}-1\right) d N_{t}-\lambda E\left(e^{x}-1\right) d t, \\
S_{0}>0, \\
d V_{t}=\kappa\left(\theta-V_{t}\right) d t+\varepsilon \sqrt{V_{t}} d B_{t}^{V}, \quad V_{0}>0,
\end{gathered}
$$

where $t \in[0, T]$ and $T>0$; on the filtered complete space $\left(\Omega, \mathscr{F},\left\{\mathscr{F}_{t}\right\}_{t \geq 0}, P\right)$, there are $B_{t}^{S}, B_{t}^{V}$ which both are 1-dimensional Brownian motions with $d B_{t}^{S} d B_{t}^{V}=\rho d t$, and $d N_{t}$ is a Poisson process with the constant jump intensity $\lambda . E[\cdot]$ is the expectation under the physical measure. The jump size $x$ is stochastic and $r, \mu, \kappa$, and $\theta$ are constants.

The integration form of stock process in (2) is given by

$$
\begin{aligned}
\ln \frac{S_{T}}{S_{t}}= & \int_{t}^{T}\left(\mu-\frac{1}{2} V_{\tau}\right) d \tau-\left[\lambda E\left(e^{x}-1\right)\right] \tau \\
& +\int_{t}^{T} \sqrt{V_{\tau}} d B_{\tau}^{S}+\sum_{i=1}^{N_{\tau}} x_{i} .
\end{aligned}
$$

We suppose that the portfolio is $(\omega, 1-\omega)$ which, respectively, means the fraction of wealth invested in the stock and money market; then the wealth process $W(t)$ and the initial wealth $W(0)=w_{0}>0$ satisfy the equations as follows:

$$
\begin{aligned}
\frac{d W_{t}}{W_{t}}= & {\left[r+\omega \phi-\omega \lambda E\left(e^{x}-1\right)\right] d t } \\
& +\omega \sqrt{V_{t}} d B_{t}^{S}+\omega\left(e^{x}-1\right) d N_{t}, \\
d V_{t}= & \kappa\left(\theta-V_{t}\right) d t+\varepsilon \sqrt{V_{t}} d B_{t}^{V},
\end{aligned}
$$

where $\phi=\mu-r$ is the equity premium.

The representative investor maximizes his/her expected utility:

$$
J(W(t), V(t), t)=\max E_{t}[U(W(T))],
$$

where $E_{t}[\cdot]$ is the conditional expectation and equals $E[\cdot$ । $\mathscr{F}_{t}$ ].

For tractability, we concentrate our attention on the case of constant relative risk aversion (CRRA) utility function:

$$
U\left(W_{T}, T\right)=\frac{W_{T}^{1-\gamma}}{1-\gamma}, \quad \gamma>0, \gamma \neq 1,
$$

where the constant $\gamma$ is the relative risk aversion coefficient.

\section{Equity Premium}

The equity premium is very important for option pricing in general equilibrium framework. Following the idea of SantaClara and Yan (2004) [12] and Zhang et al. (2012) [6], we obtain the equilibrium equity premium by modeling general equilibrium economy in the following proposition.

Proposition 1. In general equilibrium framework, the equilibrium equity premium is given by

$$
\phi=V_{t}[\gamma-\varepsilon \rho A(t)]+\lambda E\left[\left(1-e^{-\gamma x}\right)\left(e^{x}-1\right)\right],
$$

where

$$
\begin{gathered}
A(t)=\gamma(1-\gamma) \frac{1-e^{-\alpha(t-T)}}{\beta_{-}-\beta_{+} e^{-\alpha(t-T)}}, \\
\beta_{ \pm}=\kappa \pm \alpha, \quad \alpha=\sqrt{\kappa^{2}-\varepsilon^{2} \gamma(1-\gamma)} .
\end{gathered}
$$


Proof. From the optimal control problem (5), we get the Bellman equation as follows:

$$
0=J_{t}+\max _{\omega}\{\mathscr{L}(J)\}
$$

where

$$
\begin{aligned}
\mathscr{L}(J)= & {\left[r+\omega \phi-\omega \lambda E\left(e^{x}-1\right)\right] W J_{W} } \\
& +\frac{1}{2} \omega^{2} W^{2} V J_{W W}+\kappa(\theta-V) J_{V} \\
& +\frac{1}{2} \varepsilon^{2} V J_{V V}+\varepsilon \omega W V \rho J_{W V} \\
& +\lambda E\left[J\left(W\left(1+\omega\left(e^{x}-1\right)\right), V, t\right)-J\right] .
\end{aligned}
$$

Equating the derivatives of the Bellman equation (9) with respect to $\omega$ to zero, we have following equation:

$$
\begin{aligned}
0= & {\left[\phi-\lambda E\left(e^{x}-1\right)\right] W J_{W} } \\
& +\omega W^{2} V J_{W W}+\varepsilon W V \rho J_{W V} \\
& +\lambda E\left[J_{W}\left(W\left(1+\omega\left(e^{x}-1\right)\right), V, t\right) \cdot W\left(e^{x}-1\right)\right] .
\end{aligned}
$$

In equilibrium, the money market is in zero net supply. Therefore, the representative investor holds all the wealth in the stock market; that is, $\omega=1$. Then we can get the expression of $\phi$ from (11):

$$
\begin{aligned}
\phi= & -V \frac{W J_{W W}}{J_{W}}+\lambda E\left(e^{x}-1\right)-\varepsilon V \rho \frac{J_{W V}}{J_{W}} \\
& -\frac{\lambda}{J_{W}} E\left[J_{W}\left(W e^{x}, V, t\right) \cdot\left(e^{x}-1\right)\right] .
\end{aligned}
$$

Then Bellman equation (9) can be written as follows:

$$
\begin{aligned}
0= & J_{t}+r W J_{W}-\frac{1}{2} W^{2} V J_{W W}+\kappa(\theta-V) J_{V} \\
& +\frac{1}{2} \varepsilon^{2} V J_{V V}-\lambda W E\left[J_{W}\left(W e^{x}, V, t\right) \cdot\left(e^{x}-1\right)\right] \\
& +\lambda E\left[J\left(W e^{x}, V, t\right)\right]-\lambda J .
\end{aligned}
$$

From (6), we conjecture that

$$
J\left(W_{t}, V_{t}, t\right)=\exp \left(A(t) V_{t}+B(t)\right) \frac{W_{t}^{1-\gamma}}{1-\gamma} .
$$

Then, substituting (11) into (14), we obtain

$$
\begin{aligned}
0= & \dot{A}(t) V_{t}+\dot{B}(t)+r(1-\gamma)+\frac{1}{2} \gamma(1-\gamma) V \\
& +\kappa(\theta-V) A(t)+\frac{1}{2} \varepsilon^{2} V A^{2}(t) \\
& +\lambda \gamma E\left[e^{(1-\gamma) x}\right]+\lambda(1-\gamma) E\left[e^{-\gamma x}\right]-\lambda .
\end{aligned}
$$

This leads to a system of two ordinary differential equations (ODEs):

$$
\begin{gathered}
A(T)=0, \\
\dot{B}(t)+\kappa \theta A(t)+a=0, \\
B(T)=0,
\end{gathered}
$$$$
\dot{A}(t)+\frac{1}{2} \varepsilon^{2} A^{2}(t)-\kappa A(t)+\frac{1}{2} \gamma(1-\gamma)=0,
$$

where

$$
a=r(1-\gamma)+\lambda \gamma E\left[e^{(1-\gamma) x}\right]+\lambda(1-\gamma) E\left[e^{-\gamma x}\right]-\lambda .
$$

This system (16) and (17) can be solved explicitly. First, we solve the first ODE (16), which is the Riccati differential equation. Making the substitution

$$
A(t)=\frac{2}{\varepsilon^{2}} \frac{C^{\prime}(t)}{C(t)}
$$

we obtain the second-order differential equation

$$
\begin{gathered}
C^{\prime \prime}(t)-\kappa C^{\prime}(t)+\frac{\varepsilon^{2}}{4} \gamma(1-\gamma) C(t)=0, \\
C^{\prime}(T)=0 .
\end{gathered}
$$

A general solution has the form

$$
C(t)=d_{+} e^{(1 / 2) \beta_{+} t}+d_{-} e^{(1 / 2) \beta_{-} t}
$$

where

$$
\begin{gathered}
\beta_{ \pm}=\kappa \pm \alpha, \quad \alpha=\sqrt{\kappa^{2}-\varepsilon^{2} \gamma(1-\gamma)}, \\
d_{+}=\frac{\beta_{-} C(T)}{\left(\beta_{-}-\beta_{+}\right) e^{(1 / 2) \beta_{+} T}}, \quad d_{-}=\frac{\beta_{+} C(T)}{\left(\beta_{+}-\beta_{-}\right) e^{(1 / 2) \beta_{-} T}} .
\end{gathered}
$$

Thus,

$$
A(t)=\gamma(1-\gamma) \frac{1-e^{-\alpha(t-T)}}{\beta_{-}-\beta_{+} e^{-\alpha(t-T)}} .
$$

Then, the solution of the second ODE (17) is

$$
B(t)=\frac{2 \kappa \theta}{\varepsilon^{2}}\left[\frac{1}{2} \beta_{+}(t-T)+\ln \frac{\beta_{+} e^{-\alpha(t-T)}-\beta_{-}}{2 \alpha}\right]+a(t-T) .
$$

Substituting (14) into (12), we get Proposition 1.

Remark 2. In the special case where there is no stochastic volatility and jumps, $V_{t}=\sigma^{2}$ and $\varepsilon=\kappa=0$, and consequently $\phi=\sigma^{2} \gamma$ which is constant in Merton (1976) [2]. In the special case where there is no stochastic volatility, $\varepsilon=0$, and consequently $\phi=\sigma^{2} \gamma+\lambda E\left[\left(1-e^{-\gamma x}\right)\left(e^{x}-1\right)\right]$ which is constant in Zhang et al. (2012) [6]. 


\section{Option Pricing}

In this section, we will study the pricing kernel and the option pricing in general equilibrium framework. We first derive the pricing kernel which acts like the physical and risk-neutral densities in the economy and is the key to obtain the PDE of option price as follows.

Proposition 3. In general equilibrium framework, the pricing kernel is given in differential form by

$$
\begin{aligned}
\frac{d \pi_{t}}{\pi_{t}}= & -r d t-(\gamma-\varepsilon \rho A) \sqrt{V_{t}} d B_{t}^{S} \\
& +\left(e^{y}-1\right) d N_{t}-\lambda E\left(e^{y}-1\right) d t,
\end{aligned}
$$

and the integration is given by

$$
\begin{aligned}
\frac{\pi_{T}}{\pi_{t}}=\exp \{ & {\left[-r-\lambda E\left(e^{y}-1\right)\right] \tau } \\
& -\frac{1}{2} \int_{t}^{T}\left(\gamma-\varepsilon \rho A_{\tau}\right)^{2} V_{\tau} d \tau \\
& \left.-\int_{t}^{T}\left(\gamma-\varepsilon \rho A_{\tau}\right) \sqrt{V_{\tau}} d B_{\tau}^{S}+\sum_{i=1}^{N_{\tau}} y_{i}\right\},
\end{aligned}
$$

where $\tau=T-t$.

The martingale condition, $\pi_{t} S_{t}=E_{t}\left[\pi_{T} S_{T}\right]$, requires that the jump size $y$ satisfies the following restriction:

$$
E\left[\left(e^{y}-e^{-\gamma x}\right)\left(e^{x}-1\right)\right]=0 .
$$

Proof. To satisfy the martingale condition, $\pi_{t} S_{t}=E_{t}\left[\pi_{T} S_{T}\right]$, from (3) and (26), we have

$$
\begin{gathered}
E \exp \left\{\int_{t}^{T}\left[\phi_{\tau}-\frac{1}{2} V_{\tau}-\frac{1}{2}\left(\gamma-\varepsilon \rho A_{\tau}\right)^{2} V_{\tau}\right] d \tau\right. \\
-\lambda\left[E\left(e^{y}-1\right) \tau+E\left(e^{x}-1\right)\right] \tau \\
+\int_{t}^{T}\left[1-\left(\gamma-\varepsilon \rho A_{\tau}\right)\right] \sqrt{V_{\tau}} d B_{\tau}^{S} \\
\left.+\sum_{i=1}^{N_{\tau}} x_{i}+\sum_{i=1}^{N_{\tau}} y_{i}\right\}=1 .
\end{gathered}
$$

Substituting (7) into (28), we have

$$
\begin{aligned}
E \exp \left\{\int_{t}^{T} \lambda E\left[\left(1-e^{-\gamma x}\right)\left(e^{x}-1\right)\right] \tau\right. \\
-\lambda\left[E\left(e^{y}-1\right) \tau+E\left(e^{x}-1\right)\right] \tau \\
\left.\quad+\sum_{i=1}^{N_{\tau}} x_{i}+\sum_{i=1}^{N_{\tau}} y_{i}\right\}=1 .
\end{aligned}
$$

Using the property of Poisson process, $E\left[\exp \left(\sum_{i=1}^{N_{\tau}} x_{i}\right)\right]=$ $\exp \left[\lambda E\left(e^{x}-1\right) \tau\right]$, it is easy to obtain Proposition 3 .
Remark 4. In this market, there is only one tradable asset, a stock with price $S_{t}$, but there are at least two dimensions of risk, diffusive risk, and jump risk. Therefore, the market is incomplete and the pricing kernel is not unique. The nonuniqueness of the pricing kernel can be justified by the fact that the distribution of jump size $y$ in the pricing kernel can be arbitrary as long as it satisfies the martingale restriction (27). In a special case, we can choose $y=-\gamma x$, as in Liu et al. (2005) [37].

Remark 5. With Proposition 3, we define a new probability measure $Q$ :

$$
\begin{aligned}
\frac{d Q}{d P}=Z_{T}= & e^{r T} \pi_{T} \\
= & \exp \left\{-\lambda E\left(e^{y}-1\right) T-\frac{1}{2} \int_{0}^{T}\left(\gamma-\varepsilon \rho A_{\tau}\right)^{2} V_{\tau} d \tau\right. \\
& \left.-\int_{0}^{T}\left(\gamma-\varepsilon \rho A_{\tau}\right) \sqrt{V_{\tau}} d B_{\tau}^{s}+\sum_{i=1}^{N_{T}} y_{i}\right\},
\end{aligned}
$$

since, for any assets $P_{t}$ at time $t$, we have

$$
E_{t}^{\mathrm{Q}}\left[P_{T}\right]=\frac{1}{Z_{t}} E_{t}\left[Z_{T} P_{T}\right]=e^{r(T-t)} E_{t}\left[\frac{\pi_{T}}{\pi_{t}} P_{T}\right]=e^{r(T-t)} P_{t} \text {, }
$$

which means $Q$ is a risk-neutral probability measure.

Lemma 6. Define a new probability measure, $Q^{*}$, by the following Radon-Nikodym derivative:

$$
\frac{d Q^{*}}{d P}=\exp \left\{\left[-\lambda E\left(e^{y}-1\right)\right] T+\sum_{i=1}^{N_{T}} y_{i}\right\}
$$

then the following relation

$$
E\left[e^{y} f(x)\right]=E\left(e^{y}\right) E^{Q^{*}}[f(x)]
$$

is true.

Proof. The change of probability measure formula gives

$$
\begin{aligned}
E^{Q^{*}}[f(x)]= & E\left[\frac{d Q^{*}}{d P} f(x)\right] \\
= & \exp \left\{\left[-\lambda E\left(e^{y}-1\right)\right] T\right\} E\left[f(x) \prod_{i=1}^{N_{T}} e^{y_{i}}\right] \\
= & \exp \left\{\left[-\lambda E\left(e^{y}-1\right)\right] T\right\} \\
& \times \sum_{n=1}^{+\infty} e^{-\lambda T} \frac{(\lambda T)^{n}}{n !} E\left[f(x) \prod_{i=1}^{n} e^{y_{i}}\right] .
\end{aligned}
$$

Since $y_{i}, i=1,2, \ldots i, \ldots, n$, is i.i.d. and $y$ and $x$ are correlated, this means that only one of the $y_{i}$ is correlated with 
$x$. Without loss of generality, we assume that $y_{n}$ is correlated with $x$ and other $y_{i} s$ are independent of $x$. Then we have

$$
\begin{aligned}
E^{Q^{*}}[f(x)]= & E\left[\frac{d Q^{*}}{d P} f(x)\right] \\
= & \exp \left\{\left[-\lambda E\left(e^{y}-1\right)\right] T\right\} E\left[f(x) \prod_{i=1}^{N_{T}} e^{y_{i}}\right] \\
= & E\left[f(x) e^{y}\right] \exp \left\{\left[-\lambda E\left(e^{y}-1\right)\right] T\right\} \\
& \times \sum_{n=1}^{+\infty} e^{-\lambda T} \frac{(\lambda T)^{n}}{n !} E\left[f(x) \prod_{i=1}^{n-1} e^{y_{i}}\right] \\
= & \frac{E\left[e^{y} f(x)\right]}{E\left(e^{y}\right)} .
\end{aligned}
$$

Remark 7. These results are also true in $Q$ measure, because the difference between $Q$ and $Q^{*}$ is the Brownian motion that is independent of the jumps.

Now, we consider a European call written on the stock price $S_{t}$ at time $t$. The option has a payoff function $\left(S_{T}-K\right)^{+}$ at time $T$. Its price is denoted as $c\left(S_{t}, V_{t}, t\right)$ at time $t$. We derive a PDE which $c\left(S_{t}, V_{t}, t\right)$ has to satisfy in the following proposition.

Proposition 8. In general equilibrium framework, the price of European call option satisfies the following PDE:

$$
\begin{gathered}
0=c_{t}+\frac{1}{2} V S^{2} c_{S S}+\frac{1}{2} \varepsilon^{2} V c_{V V}+\varepsilon \rho V S c_{S V} \\
+\left[r-\lambda^{Q} E^{Q}\left(e^{x}-1\right)\right] S c_{S}-r c+\left[\kappa^{Q}\left(\theta^{Q}-V\right)\right] c_{V} \\
+\lambda^{Q}\left\{E^{Q}\left[c\left(S e^{x}, V, t\right)\right]-c(S, V, t)\right\}, \\
c(S, V, T)=(S-K)^{+},
\end{gathered}
$$

where

$$
\begin{gathered}
E^{Q}[f(x)]=\frac{E\left[e^{y} f(x)\right]}{E\left(e^{y}\right)}, \quad \lambda^{Q}=\lambda E\left(e^{y}\right), \\
\kappa^{Q}=\kappa+(\gamma-\varepsilon \rho A) \varepsilon \rho, \quad \theta^{Q}=\frac{\kappa \theta}{\kappa+(\gamma-\varepsilon \rho A) \varepsilon \rho} .
\end{gathered}
$$

Proof. First, we rewrite the stock with continue part and jump part:

$$
d S=d^{C} S+S\left(e^{x}-1\right) d N_{t}
$$

where

$$
\begin{gathered}
d^{C} S=\left[r+\phi-\lambda E\left(e^{x}-1\right)\right] S d t+\sqrt{V} S d B^{S} \\
d V=\kappa(\theta-V) d t+\varepsilon \sqrt{V} d B_{t}^{V} .
\end{gathered}
$$

Similarly,

$$
d \pi=d^{C} \pi+\left(e^{y}-1\right) \pi d N_{t},
$$

where

$$
\begin{gathered}
d^{C} \pi=\left[-r-\lambda E\left(e^{y}-1\right)\right] \pi d t-(\gamma-\varepsilon \rho A) \sqrt{V} \pi d B_{t}^{S}, \\
d c=d^{C} c+\left[c\left(S e^{x}, V, t\right)-c(S, V, t)\right] d N_{t},
\end{gathered}
$$

where

$$
\begin{aligned}
d^{C} c= & \left\{c_{t}+\left(r+\phi-\lambda E\left(e^{x}-1\right)\right) S c_{S}+\frac{1}{2} V S^{2} c_{S S}\right. \\
& \left.+\kappa(\theta-V) c_{V}+\frac{1}{2} \varepsilon^{2} V c_{V V}+c_{S V} \varepsilon \rho S V\right\} d t \\
& +\sqrt{V} S c_{S} d B^{S}+\varepsilon \sqrt{V} c_{V} d B_{t}^{V} .
\end{aligned}
$$

From (40) and (42), we get

$$
d(\pi c)=d^{C}(\pi c)+\left[\pi e^{y} c\left(S e^{x}, V, t\right)-\pi c(S, V, t)\right] d N_{t},
$$

where

$$
\begin{aligned}
d^{C}(\pi c)= & c\left[-r-\lambda E\left(e^{y}-1\right)\right] \pi d t \\
& -c(\gamma-\varepsilon \rho A) \sqrt{V} \pi d B_{t}^{S} \\
+ & \pi\left\{c_{t}+\left(r+\phi-\lambda E\left(e^{x}-1\right)\right) S c_{S}+\frac{1}{2} V S^{2} c_{S S}\right. \\
& \left.+\kappa(\theta-V) c_{V}+\frac{1}{2} \varepsilon^{2} V c_{V V}+c_{S V} \varepsilon \rho S\right\} d t \\
& +\pi \sqrt{V} S c_{S} d B^{S}+\pi \varepsilon \sqrt{V} c_{V} d B_{t}^{V} \\
& -V \pi(\gamma-\varepsilon \rho A)\left(S c_{S} d t+\varepsilon \rho c_{V} d t\right) .
\end{aligned}
$$

The martingale condition $E[d(\pi c)]=0$ requires

$$
\begin{aligned}
0= & c_{t}+\frac{1}{2} V S^{2} c_{S S}+\frac{1}{2} \varepsilon^{2} V c_{V V} \\
& +\left[\left(r+\phi-V(\gamma-\varepsilon \rho A)-\lambda E\left(e^{x}-1\right)\right)\right] S c_{S} \\
& -\left[r+\lambda E\left(e^{y}-1\right)\right] c \\
& +[\kappa(\theta-V)-V(\gamma-\varepsilon \rho A) \varepsilon \rho] c_{V} \\
& +\varepsilon \rho S V c_{S V}+\lambda E\left[e^{y} c\left(S e^{x}, V, t\right)\right]-\lambda c(S, V, t) .
\end{aligned}
$$

Using Lemma 6, we have

$$
E^{Q}[f(x)]=\frac{E\left[e^{y} f(x)\right]}{E\left(e^{y}\right)} .
$$

Denoting $\lambda^{\mathrm{Q}}=\lambda E\left(e^{y}\right)$ and using the restriction condition $E\left[\left(e^{y}-e^{-\gamma x}\right)\left(e^{x}-1\right)\right]=0, \phi=V[\gamma-\varepsilon \rho A]+\lambda E\left[\left(1-e^{-\gamma x}\right)\left(e^{x}-1\right)\right]$ 
in Proposition 1 and the terminal payoff function $c(S, V, T)=$ $(S-K)^{+}$, we obtain

$$
\begin{gathered}
0=c_{t}+\frac{1}{2} V S^{2} c_{S S}+\frac{1}{2} \varepsilon^{2} V c_{V V}+\varepsilon \rho V S c_{S V} \\
+\left[r-\lambda^{Q} E^{Q}\left(e^{x}-1\right)\right] S c_{S}-r c \\
+[\kappa(\theta-V)-V(\gamma-\varepsilon \rho A) \varepsilon \rho] c_{V} \\
+\lambda^{Q}\left\{E^{Q}\left[c\left(S e^{x}, V, t\right)\right]-c(S, V, t)\right\} \\
c(S, V, T)=(S-K)^{+} .
\end{gathered}
$$

If we denote $\kappa^{\mathrm{Q}}=\kappa+(\gamma-\varepsilon \rho A) \varepsilon \rho$ and $\theta^{\mathrm{Q}}=(\kappa \theta /(\kappa+(\gamma-$ $\varepsilon \rho A) \varepsilon \rho)$ ), then (48) can be written as

$$
\begin{gathered}
0=c_{t}+\frac{1}{2} V S^{2} c_{S S}+\frac{1}{2} \varepsilon^{2} V c_{V V}+\varepsilon \rho V S c_{S V} \\
+\left[r-\lambda^{Q} E^{Q}\left(e^{x}-1\right)\right] S c_{S} \\
-r c+\left[\kappa^{Q}\left(\theta^{Q}-V\right)\right] c_{V} \\
+\lambda^{Q}\left\{E^{Q}\left[c\left(S e^{x}, V, t\right)\right]-c(S, V, t)\right\} \\
c(S, V, T)=(S-K)^{+} .
\end{gathered}
$$

Remark 9. The stock process (2) in a risk-neutral measure $Q$ can be written as

$$
\begin{gathered}
\frac{d S_{t}}{S_{t}}=r d t+\sqrt{V_{t}} d \widetilde{B}_{t}^{S}+\left(e^{x}-1\right) d N_{t}-\lambda^{\mathrm{Q}} E^{\mathrm{Q}}\left(e^{x}-1\right) d t, \\
S_{0}>0, \\
d V_{t}=\kappa^{\mathrm{Q}}\left(\theta^{\mathrm{Q}}-V_{t}\right) d t+\varepsilon \sqrt{V_{t}} d \widetilde{B}_{t}^{V}, \quad V_{0}>0,
\end{gathered}
$$

where

$$
\begin{gathered}
d \widetilde{B}_{t}^{S}=d B_{t}^{S}+(\gamma-\varepsilon \rho A) \sqrt{V_{t}} d t, \\
d \widetilde{B}_{t}^{V}=d B_{t}^{V}+\rho(\gamma-\varepsilon \rho A) \sqrt{V_{t}} d t \\
\lambda^{Q}=\lambda E\left(e^{y}\right), \quad \kappa^{Q}=\kappa+(\gamma-\varepsilon \rho A) \varepsilon \rho, \\
\theta^{Q}=\frac{\kappa \theta}{\kappa+(\gamma-\varepsilon \rho A) \varepsilon \rho}, \quad E^{Q}[f(x)]=\frac{E\left[e^{y} f(x)\right]}{E\left(e^{y}\right)} .
\end{gathered}
$$

The proof is very easy. Substituting all equations in Remark 9 to (50) and using the equilibrium equity premium (7) and the restriction (27), we will get (2).

Furthermore, we also can understand $\lambda^{\mathrm{Q}}=\lambda E\left(e^{y}\right)$ by

$$
\begin{aligned}
E_{0}^{\mathrm{Q}}\left[N_{T}\right] & =E_{0}\left[Z_{T} N_{T}\right]=E_{0}\left[e^{-\lambda E\left(e^{y}-1\right) T+\sum_{i=1}^{N_{T}} y_{i}} N_{T}\right] \\
& =\sum_{n=0}^{+\infty} e^{-\lambda E\left(e^{y}\right) T} \frac{\left(\lambda E\left(e^{y}\right) T\right)^{n}}{n !} n=\lambda E\left(e^{y}\right) T:=\lambda^{Q} T .
\end{aligned}
$$

Employing the Feynman-Kac theorem to (38), we also can obtain PDE (36) in Proposition 8.

Remark 10. $\kappa^{\mathrm{Q}}=\kappa+(\gamma-\varepsilon \rho A) \varepsilon \rho$ and $\theta^{\mathrm{Q}}=(\kappa \theta /(\kappa+(\gamma-$ $\varepsilon \rho A) \varepsilon \rho)$ ) are similar forms in Heston (1993) [40].

Following the approach of Lewis (2000) [8], we will derive the pricing formula in a risk-neutral measure $Q$ from the PDE.

Proposition 11. In general equilibrium framework, the pricing formula is given by

$$
\begin{aligned}
& c(S, V, \tau) \\
& \quad=S-\frac{e^{-r \tau}}{2 \pi} \int_{(i / 2)-\infty}^{(i / 2)+\infty} \frac{K^{i k+1}}{k^{2}-i k} e^{-i k(r \tau+\ln S)+\Gamma^{\mathrm{Q}}(\tau) V+\Lambda^{\mathrm{Q}}(\tau)} d k,
\end{aligned}
$$

where

$$
\begin{gathered}
\Gamma^{\mathrm{Q}}(\tau)=\left(k^{2}-i k\right) \frac{1-e^{-\zeta^{\mathrm{Q}} \tau}}{\xi_{-}^{\mathrm{Q}}-\xi_{+}^{\mathrm{Q}} e^{-\zeta^{\mathrm{Q}} \tau}}, \\
\Lambda^{\mathrm{Q}}(\tau)=-\frac{2 \kappa^{\mathrm{Q}} \theta^{\mathrm{Q}}}{\varepsilon^{2}}\left[\frac{1}{2} \xi_{+}^{\mathrm{Q}} \tau+\ln \left(\frac{\xi_{+}^{\mathrm{Q}} e^{-\zeta^{\mathrm{Q}} \tau}-\xi_{-}^{\mathrm{Q}}}{2 \zeta^{\mathrm{Q}}}\right)\right]+b^{\mathrm{Q}} \tau \\
\xi_{ \pm}^{\mathrm{Q}}=\left(i k \varepsilon \rho+\kappa^{\mathrm{Q}}\right) \pm \zeta^{\mathrm{Q}} \\
\zeta^{\mathrm{Q}}=\sqrt{\left(i k \varepsilon \rho+\kappa^{\mathrm{Q}}\right)^{2}+\varepsilon^{2}\left(k^{2}-i k\right)}, \\
b^{\mathrm{Q}}=i k \lambda^{\mathrm{Q}} E^{\mathrm{Q}}\left(e^{x}-1\right)+\lambda^{\mathrm{Q}} E^{\mathrm{Q}}\left(e^{-i k x}-1\right) .
\end{gathered}
$$

Proof. We denote $X=\ln S$ and $g(X, V, t):=c\left(e^{X}, V, t\right)=$ $c(S, V, t)$; then, the PDE (36) can be rewritten as

$$
\begin{aligned}
&-g_{t}=-r g+\frac{1}{2} V g_{X X}+\frac{1}{2} \varepsilon^{2} V g_{V V}+\varepsilon \rho V g_{X V} \\
&+\left[r-\lambda^{Q} E^{Q}\left(e^{x}-1\right)-\frac{1}{2} V\right] g_{X}+\left[\kappa^{Q}\left(\theta^{Q}-V\right)\right] g_{V} \\
&+\lambda^{Q}\left\{E^{Q}[g(X+x, V, t)]-g(X, V, t)\right\} \\
& g(X, V, T)=\left(e^{X}-K\right)^{+}
\end{aligned}
$$

Let $G(k, V, t)$ be Fourier transform of $g(X, V, t)$ :

$$
G(k, V, t)=\int_{-\infty}^{+\infty} e^{i k X} g(X, V, t) d X
$$

with $G(k, V, T)=-\left(K^{i k+1} /\left(k^{2}-i k\right)\right)$. 

by

Denote $k=k_{r}+i k_{i}$; the inverse Fourier transform is given

$$
g(X, V, t)=\frac{1}{2 \pi} \int_{i k_{i}-\infty}^{i k_{i}+\infty} e^{-i k X} G(k, V, t) d k .
$$

Then, the PDE (55) can be rewritten as

$$
\begin{aligned}
&-G_{t}=-(1+i k) r G+i k \lambda^{Q} E^{Q}\left(e^{x}-1\right) G-\frac{1}{2} V\left(k^{2}-i k\right) G \\
&+\frac{1}{2} \varepsilon^{2} V G_{V V}-i k \varepsilon \rho V G_{V} \\
&+\left[\kappa^{Q}\left(\theta^{Q}-V\right)\right] G_{V}+\lambda^{Q} E^{Q}\left[e^{-i k x}-1\right] G, \\
& G(k, V, T)=-\frac{K^{i k+1}}{k^{2}-i k} .
\end{aligned}
$$

Denote $h \equiv e^{(1+i k) r \tau} G$; the PDE (58) can be rewritten as

$$
\begin{aligned}
& h_{\tau}= {\left[i k \lambda^{Q} E^{Q}\left(e^{x}-1\right)-\frac{1}{2} V\left(k^{2}-i k\right)\right] h } \\
&+\frac{1}{2} \varepsilon^{2} V h_{V V}+\left[\kappa^{Q}\left(\theta^{Q}-V\right)-i k \varepsilon \rho V\right] h_{V} \\
&+\lambda^{Q} E^{Q}\left[e^{-i k x}-1\right] h \\
& \text { with } h(k, V, 0)=-\frac{K^{i k+1}}{k^{2}-i k} .
\end{aligned}
$$

According to the study of Lewis (2000) [8], to solve the PDE (59) with the initial condition (60), it is enough to solve the same equation with the initial value being equal to one. We call this solution fundamental transform and denote it $h(k, V, \tau)$ which satisfies following equation by:

$$
\begin{gathered}
h_{\tau}=\left[i k \lambda^{\mathrm{Q}} E^{\mathrm{Q}}\left(e^{x}-1\right)-\frac{1}{2} V\left(k^{2}-i k\right)\right] h+\frac{1}{2} \varepsilon^{2} V h_{V V} \\
+\left[\kappa^{\mathrm{Q}}\left(\theta^{\mathrm{Q}}-V\right)-i k \varepsilon \rho V\right] h_{V}+\lambda^{\mathrm{Q}} E^{\mathrm{Q}}\left[e^{-i k x}-1\right] h, \\
h(k, V, 0)=1,
\end{gathered}
$$

and the option price satisfies

$$
\begin{aligned}
& c(S, V, \tau) \\
& \quad=S-\frac{1}{2 \pi} \int_{(i / 2)-\infty}^{(i / 2)+\infty} e^{-i k \ln S} e^{-(1+i k) r \tau} \frac{K^{i k+1}}{k^{2}-i k} h(k, V, \tau) d k .
\end{aligned}
$$

Denoting $H(V, \tau):=h(k, V, \tau)$, then, the PDE (61) can be rewritten as

$$
\begin{gathered}
H_{\tau}=\left[i k \lambda^{\mathrm{Q}} E^{\mathrm{Q}}\left(e^{x}-1\right)-\frac{1}{2} V\left(k^{2}-i k\right)\right] H+\frac{1}{2} \varepsilon^{2} V H_{V V} \\
+\left[\kappa^{\mathrm{Q}}\left(\theta^{\mathrm{Q}}-V\right)-i k \varepsilon \rho V\right] H_{V}+\lambda^{\mathrm{Q}} E^{\mathrm{Q}}\left[e^{-i k x}-1\right] H, \\
H(V, 0)=1 .
\end{gathered}
$$

We guess that a solution of (63) is as the following form:

$$
H(V, \tau)=e^{\Gamma^{\mathrm{Q}}(\tau) V+\Lambda^{\mathrm{Q}}(\tau)},
$$

with $\Gamma^{\mathrm{Q}}(0)=0$ and $\Lambda^{\mathrm{Q}}(0)=0$.

This leads to a system of two ODEs:

$$
\begin{gathered}
\dot{\Gamma}^{\mathrm{Q}}(\tau)-\frac{1}{2} \varepsilon^{2}\left(\Gamma^{\mathrm{Q}}(\tau)\right)^{2}+\left(i k \varepsilon \rho+\kappa^{\mathrm{Q}}\right) \Gamma^{\mathrm{Q}}(\tau) \\
+\frac{1}{2}\left(k^{2}-i k\right)=0, \\
\Gamma^{\mathrm{Q}}(0)=0, \\
\dot{\Lambda}^{\mathrm{Q}}(\tau)-\kappa^{\mathrm{Q}} \theta^{\mathrm{Q}} \Gamma^{\mathrm{Q}}(\tau)-b^{\mathrm{Q}}=0, \\
\Lambda^{\mathrm{Q}}(0)=0,
\end{gathered}
$$

where $b^{\mathrm{Q}}=i k \lambda^{\mathrm{Q}} E^{\mathrm{Q}}\left(e^{x}-1\right)+\lambda^{\mathrm{Q}} E^{\mathrm{Q}}\left(e^{-i k x}-1\right)$.

This system can be solved explicitly. First, we solve the first ODE (65), which is the Riccati differential equation. Making the substitution

$$
\Gamma^{\mathrm{Q}}(\tau)=-\frac{2}{\varepsilon^{2}} \frac{I^{\prime}(\tau)}{I(\tau)}
$$

we obtain the second-order differential equation

$$
\begin{gathered}
I^{\prime \prime}(\tau)+\left(i k \varepsilon \rho+\kappa^{Q}\right) I^{\prime}(\tau)-\frac{\varepsilon^{2}}{4}\left(k^{2}-i k\right) I(\tau)=0, \\
I^{\prime}(0)=0 .
\end{gathered}
$$

A general solution of (68) has the form

$$
I(\tau)=D_{+}^{Q} e^{(1 / 2) \xi_{+}^{\mathrm{Q}} \tau}+D_{-}^{\mathrm{Q}} e^{(1 / 2) \xi_{-}^{\mathrm{Q}} \tau}
$$

where

$$
\xi_{ \pm}^{\mathrm{Q}}=\left(i k \varepsilon \rho+\kappa^{\mathrm{Q}}\right) \pm \zeta^{\mathrm{Q}}
$$

$$
\begin{aligned}
\zeta^{\mathrm{Q}} & =\sqrt{\left(i k \varepsilon \rho+\kappa^{\mathrm{Q}}\right)^{2}+\varepsilon^{2}\left(k^{2}-i k\right)} \\
D_{+}^{\mathrm{Q}} & =\frac{\xi_{-}^{\mathrm{Q}} I(0)}{\xi_{-}^{\mathrm{Q}}-\xi_{+}^{\mathrm{Q}}}, \quad D_{-}^{\mathrm{Q}}=\frac{\xi_{+}^{\mathrm{Q}} I(0)}{\xi_{+}^{\mathrm{Q}}-\xi_{-}^{\mathrm{Q}}} .
\end{aligned}
$$

Thus,

$$
\Gamma^{\mathrm{Q}}(\tau)=\left(k^{2}-i k\right) \frac{1-e^{-\zeta^{\mathrm{Q}} \tau}}{\xi_{-}^{\mathrm{Q}}-\xi_{+}^{\mathrm{Q}} e^{-\zeta^{\mathrm{Q} \tau}}} .
$$

Then, the solution of the second $\operatorname{ODE}(66)$ is

$$
\Lambda^{\mathrm{Q}}(\tau)=-\frac{2 \kappa^{\mathrm{Q}} \theta^{\mathrm{Q}}}{\varepsilon^{2}}\left[\frac{1}{2} \xi_{+}^{\mathrm{Q}} \tau+\ln \left(\frac{\xi_{+}^{\mathrm{Q}} e^{-\zeta^{\mathrm{Q}} \tau}-\xi_{-}^{\mathrm{Q}}}{2 \zeta^{\mathrm{Q}}}\right)\right]+b^{\mathrm{Q}} \tau
$$


Note. Although the pricing formula (40) contains a complex integral, the result is real.

With the European option price being well understood, we study the relations between the physical and risk-neutral densities and moments which can help us to study the negative variance risk premium, the implied volatility smirk, and the prediction of realized skewness. In this paper, we extend the results about those relations in [6] to a stochastic volatility case.

Proposition 12. The risk-neutral density of the continuously compounded return within $\tau=T-t, R_{\tau}=\ln S_{T} / S_{t}, S_{t}$ given in (38), is given by

$$
\varphi^{\mathrm{Q}}\left(R_{\tau} \mid V, \tau\right)=\frac{e^{-r \tau}}{2 \pi} \int_{i(i / 2)-\infty}^{(i / 2)+\infty} e^{-i k(R-r \tau)+\Gamma^{\mathrm{Q}}(\tau) V+\Lambda^{\mathrm{Q}}(\tau)} d k .
$$

The first moment, second, third, and fourth central moments of the continuously compounded return in the risk-neutral measure are given by

$$
\begin{gathered}
E_{t}^{\mathrm{Q}}\left(R_{\tau}\right)=\left[\mu-\frac{1}{2} E_{t}^{\mathrm{Q}}\left(V_{\tau}\right)-\lambda^{\mathrm{Q}} E^{\mathrm{Q}}\left(e^{x}-1-x\right)\right] \tau \\
E_{t}^{\mathrm{Q}}\left[R_{\tau}-E_{t}^{\mathrm{Q}}\left(R_{\tau}\right)\right]^{2}=\tau E_{t}^{\mathrm{Q}}\left(V_{\tau}\right)+\lambda^{\mathrm{Q}} \tau\left[\left(\mu_{x}^{\mathrm{Q}}\right)^{2}+\operatorname{Var}^{\mathrm{Q}}(x)\right] \\
E_{t}^{\mathrm{Q}}\left[R_{\tau}-E_{t}^{\mathrm{Q}}\left(R_{\tau}\right)\right]^{3} \\
=\lambda^{\mathrm{Q}} \tau\left[\left(\mu_{x}^{\mathrm{Q}}\right)^{3}+3 \mu_{x}^{\mathrm{Q}} \operatorname{Var}^{\mathrm{Q}}(x)+3 r d^{\mathrm{Q}}(x)\right] \\
E_{t}^{\mathrm{Q}}\left[R_{\tau}-E_{t}^{\mathrm{Q}}\left(R_{\tau}\right)\right]^{4} \\
=3\left(\lambda^{\mathrm{Q}} \tau\left[\left(\mu_{x}^{\mathrm{Q}}\right)^{2}+\operatorname{Var}^{\mathrm{Q}}(x)\right]+\tau E_{t}^{\mathrm{Q}}\left[V_{\tau}\right]\right)^{2} \\
+\lambda^{\mathrm{Q}} \tau\left[\left(\mu_{x}^{\mathrm{Q}}\right)^{4}+6\left(\mu_{x}^{\mathrm{Q}}\right)^{2} \operatorname{Var}^{\mathrm{Q}}(x)\right. \\
\left.+4 \mu_{x}^{\mathrm{Q}} 3 r d^{\mathrm{Q}}(x)+4 t h^{\mathrm{Q}}(x)\right]
\end{gathered}
$$

where $E_{t}^{\mathrm{Q}}\left(V_{\tau}\right)=e^{-\kappa^{\mathrm{Q}} \tau} V_{0}+\theta^{\mathrm{Q}}\left(1-e^{-\kappa^{\mathrm{Q}} \tau}\right), \mu_{x}^{\mathrm{Q}}=E^{\mathrm{Q}}(x)$ is the first moment, $\operatorname{Var}^{\mathrm{Q}}(x)=E^{\mathrm{Q}}\left[\left(x-\mu_{x}^{\mathrm{Q}}\right)^{2}\right], 3 r d^{\mathrm{Q}}(x)=E^{\mathrm{Q}}[(x-$ $\left.\left.\mu_{x}^{\mathrm{Q}}\right)^{3}\right]$, and $4 t^{\mathrm{Q}}(x)=E^{\mathrm{Q}}\left[\left(x-\mu_{x}^{\mathrm{Q}}\right)^{4}\right]$ are the second, third, and fourth central moments in the risk-neutral measure of random number $x$.

The physical density is given by

$$
\varphi\left(R_{\tau} \mid V, \tau\right)=\frac{e^{-\mu \tau}}{2 \pi} \int_{(i / 2)-\infty}^{(i / 2)+\infty} e^{-i k(R-\mu \tau)+\Gamma(\tau) V+\Lambda(\tau)} d k,
$$

where

$$
\begin{gathered}
\Gamma(\tau)=\left(k^{2}-i k\right) \frac{1-e^{-\zeta \tau}}{\xi_{-}-\xi_{+} e^{-\zeta \tau}} \\
\Lambda(\tau)=-\frac{2 \kappa \theta}{\varepsilon^{2}}\left[\frac{1}{2} \xi_{+} \tau+\ln \left(\frac{\xi_{+} e^{-\zeta \tau}-\xi_{-}}{2 \zeta}\right)\right]+b \tau,
\end{gathered}
$$

$$
\begin{gathered}
\xi_{ \pm}=(i k \varepsilon \rho+\kappa) \pm \zeta, \\
\zeta=\sqrt{(i k \varepsilon \rho+\kappa)^{2}+\varepsilon^{2}\left(k^{2}-i k\right),} \\
b=i k \lambda E\left(e^{x}-1\right)+\lambda E\left(e^{-i k x}-1\right) .
\end{gathered}
$$

The first moment, second, third, and fourth central moments of the continuously compounded return in the physical measure are given by

$$
\begin{array}{r}
E_{t}\left(R_{\tau}\right)=\left[\mu-\frac{1}{2} E_{t}\left(V_{\tau}\right)-\lambda E\left(e^{x}-1-x\right)\right] \tau, \\
E_{t}\left[R_{\tau}-E_{t}\left(R_{\tau}\right)\right]^{2}=\tau E_{t}\left(V_{\tau}\right)+\lambda \tau\left[\mu_{x}^{2}+\operatorname{Var}(x)\right], \\
E_{t}\left[R_{\tau}-E_{t}\left(R_{\tau}\right)\right]^{3}=\lambda \tau\left[\mu_{x}^{3}+3 \mu_{x} \operatorname{Var}(x)+3 r d(x)\right], \\
E_{t}\left[R_{\tau}-E_{t}\left(R_{\tau}\right)\right]^{4}=3\left(\lambda \tau\left[\mu_{x}^{2}+\operatorname{Var}(x)\right]+\tau E_{t}\left[V_{\tau}\right]\right)^{2} \\
+\lambda \tau\left[\mu_{x}^{4}+6 \mu_{x}^{2} \operatorname{Var}(x)\right. \\
\left.+4 \mu_{x} 3 r d(x)+4 t h(x)\right],
\end{array}
$$

where $E_{t}\left(V_{\tau}\right)=e^{-\kappa \tau} V_{0}+\theta\left(1-e^{-\kappa \tau}\right), \mu_{x}=E(x)$ is the first moment, $\operatorname{Var}(x)=E\left[\left(x-\mu_{x}\right)^{2}\right], 3 r d(x)=E\left[\left(x-\mu_{x}\right)^{3}\right]$, and $4 t h(x)=E\left[\left(x-\mu_{x}\right)^{4}\right]$ are second, third, and fourth central moments in the physical measure of random number $x$.

Proof. We denote the corresponding probability function $\Phi=\operatorname{Prob}\left(S_{T} \leq K\right)$, and $\Phi$ also satisfies the $\operatorname{PDE}(36)$ with a different boundary condition $\left.\Phi\right|_{t=T}=1_{\left\{S_{T} \leq K\right\}}$. Under the Fourier transform, this boundary condition becomes $\left(K^{i k} / i k\right)$. Solving the PDE (36) under this new boundary condition, we have

$$
\Phi=\frac{e^{-r \tau}}{2 \pi} \int_{i(i / 2)-\infty}^{(i / 2)+\infty} \frac{e^{i k(\ln (K / S)-r \tau)}}{i k} e^{\Gamma^{Q}(\tau) V+\Lambda^{Q}(\tau)} d k .
$$

We denote the stock return between time $t$ and $T$ by $R$ so that $K=S e^{R}$; then we can differentiate $\Phi$ with respect to $R$ to get the density function $\varphi^{\mathrm{Q}}\left(R_{\tau} \mid V, \tau\right)$. We find that the parameters of the physical stock price process in (2) are the same those of risk-neutral stock price process in (50); then the parameters of $\varphi\left(R_{\tau} \mid V, \tau\right)$ are just removed by the superscript Q .

Now we compute the first moment, second, third, and fourth central moments of the continuously compounded return in the physical measure. There are at least two methods to solve the problem of how to compute central moments.

One method is using the $R_{\tau}$ 's characteristic function to calculate the $n$th moment of $R_{\tau}$. Since

$$
\begin{gathered}
f(k, V, t)=\int_{-\infty}^{+\infty} e^{i k R} \varphi(R \mid V, \tau) d R, \\
\frac{\partial^{n} f}{\partial k^{n}}=(i)^{n} \int_{-\infty}^{+\infty} R^{n} e^{i k R} \varphi(R \mid V, \tau) d R, \\
E_{t}\left(R_{\tau}^{n}\right)=\left.(-i)^{n} \frac{\partial^{n} f}{\partial k^{n}}\right|_{k=0},
\end{gathered}
$$


then

$$
E_{t}\left(R_{\tau}^{n}\right)=\left.\int_{-\infty}^{+\infty} R^{n} e^{i k R} \varphi(R \mid V, \tau) d R\right|_{k=0} .
$$

Although this method is very straightforward, it is too complicated to calculate the integration. We introduce another way to obtain the central moments by a direct calculation.

From (3), we have

$$
R_{\tau}=\left[\mu-\lambda E\left(e^{x}-1\right)\right] \tau-\frac{1}{2} \int_{t}^{T} V_{\tau} d \tau+\int_{t}^{T} \sqrt{V_{\tau}} d B_{\tau}^{S}+\sum_{i=1}^{N_{\tau}} x_{i} .
$$

Then, the first moment of the continuously compounded return in the physical measure can be easy to be obtained:

$$
\begin{aligned}
E_{t}\left(R_{\tau}\right)= & {\left[\mu-\lambda E\left(e^{x}-1-x\right)\right] \tau } \\
& -\frac{1}{2} \int_{t}^{T} E_{t}\left[V_{\tau}\right] d \tau \\
= & {\left[\mu-\frac{1}{2} E_{t}\left[V_{\tau}\right]-\lambda E\left(e^{x}-1-x\right)\right] \tau . }
\end{aligned}
$$

Define the first central moment, second, third, and fourth central moments in the physical measure of random number $x$ as $\mu_{x}=E(x), \operatorname{Var}(x)=E\left[\left(x-\mu_{x}\right)^{2}\right], 3 \operatorname{rd}(x)=E\left[\left(x-\mu_{x}\right)^{3}\right]$, and $4 \operatorname{th}(x)=E\left[\left(x-\mu_{x}\right)^{4}\right]$.

From (2) we have

$$
d\left(e^{\kappa \tau} V_{\tau}\right)=\kappa \theta e^{\kappa \tau} d t+\varepsilon e^{\kappa \tau} \sqrt{V_{\tau}} d B_{\tau}^{V} .
$$

The integration is given by

$$
e^{\kappa \tau} V_{\tau}=V_{0}+\theta\left(e^{\kappa \tau}-1\right) \tau+\varepsilon \int_{0}^{\tau} e^{\kappa \tau} \sqrt{V_{\tau}} d B_{\tau}^{V} .
$$

Then

$$
E_{t}\left(V_{\tau}\right)=e^{-\kappa \tau} V_{0}+\theta\left(1-e^{-\kappa \tau}\right) .
$$

Using (81), we have

$$
\begin{aligned}
E_{t}\left[R_{\tau}-E_{t}\left(R_{\tau}\right)\right]^{2}= & E_{t}\left[\int_{t}^{T} \sqrt{V_{\tau}} d B_{\tau}^{S} \cdot \int_{t}^{T} \sqrt{V_{\tau}} d B_{\tau}^{S}\right] \\
& +E_{t}\left[\left(\sum_{i=1}^{N_{\tau}} x_{i}-\lambda \tau \mu_{x}\right)^{2}\right] \\
= & \int_{t}^{T} E_{t}\left[V_{\tau}\right] d \tau+E\left(N_{\tau}\right) E\left[\left(x-\mu_{x}\right)^{2}\right] \\
& +2 E\left[N_{\tau}\left(N_{\tau}-\lambda \tau\right)\right] E\left(x-\mu_{x}\right) \mu_{x} \\
& +E\left[\left(x-\mu_{x}\right)^{2}\right] \mu_{x}^{2} \\
= & E_{t}\left[V_{\tau}\right] \tau+\lambda \tau\left[\mu_{x}^{2}+\operatorname{Var}(x)\right],
\end{aligned}
$$

$$
\begin{aligned}
E_{t}\left[R_{\tau}-E_{t}\left(R_{\tau}\right)\right]^{3}= & E_{t}\left[\left(\sum_{i=1}^{N_{\tau}} x_{i}-\lambda \tau \mu_{x}\right)^{3}\right] \\
= & \lambda \tau\left[\mu_{x}^{3}+3 \mu_{x} \operatorname{Var}(x)+3 \operatorname{rd}(x)\right] \\
E_{t}\left[R_{\tau}-E_{t}\left(R_{\tau}\right)\right]^{4}= & 3 ! !\left(E_{t}\left[\int_{t}^{T} V_{\tau} d \tau\right]\right)^{2} \\
& +6\left[\int_{t}^{T} E_{t}\left[V_{\tau}\right] d \tau\right] \\
& \cdot E_{t}\left[\left(\sum_{i=1}^{N_{\tau}} x_{i}-\lambda \tau \mu_{x}\right)^{2}\right] \\
& +E_{t}\left[\left(\sum_{i=1}^{N_{\tau}} x_{i}-\lambda \tau \mu_{x}\right)^{4}\right] \\
= & 3 \tau^{2}\left(E_{t}\left[V_{\tau}\right]\right)^{2} \\
& +6 \lambda \tau E_{t}\left[V_{\tau}\right]\left(\mu_{x}^{2}+\operatorname{Var}(x)\right) \\
& +\lambda \tau\left[\mu_{x}^{4}+6 \mu_{x}^{2} \operatorname{Var}(x)\right. \\
& \left.+4 \mu_{x} 3 \operatorname{rrd}(x)+4 \operatorname{th}(x)\right] \\
& +3 \lambda^{2} \tau^{2}\left[\mu_{x}^{2}+\operatorname{Var}(x)\right]^{2} \\
= & 3\left(\lambda \tau\left[\mu_{x}^{2}+\operatorname{Var}(x)\right]+\tau E_{t}\left[V_{\tau}\right]\right)^{2} \\
& +\lambda \tau\left[\mu_{x}^{4}+6 \mu_{x}^{2} \operatorname{Var}(x)\right. \\
& \left.+4 \mu_{x} 3 \operatorname{rd}(x)+4 \operatorname{th}(x)\right]
\end{aligned}
$$

The results of risk-neutral moments can be obtained with the same procedure in the risk-neutral probability measure.

Remark 13. If $E_{t}\left[V_{\tau}\right]=E_{t}^{\mathrm{Q}}\left[V_{\tau}\right]=\sigma^{2}$, where $\sigma^{2}$ is constant, our results will degenerate into Proposition 3.19 by Zhang et al. (2012) [6].

We denote $\overline{\text { Skewness, }}, \overline{\text { Kurtosis }}$ as the skewness and kurtosis in risk-neutral measure, respectively. Skewness and kurtosis are very important for asset pricing. For example, Bakshi et al. (2003) [13] concluded that variations in the risk-neutral skew were instrumental in explaining the differential pricing of individual equity options and found that less negatively skewed stocks have flatter smiles. From Proposition 12, we easily obtain the following three corollaries about skewness and kurtosis.

Corollary 14. The skewness and kurtosis in physical measure are given by

$$
\begin{aligned}
\text { Skewness } & =\frac{E_{t}\left[R_{\tau}-E_{t}\left(R_{\tau}\right)\right]^{3}}{\left[E_{t}\left[R_{\tau}-E_{t}\left(R_{\tau}\right)\right]^{2}\right]^{3 / 2}} \\
& =\frac{\lambda \tau\left[\mu_{x}^{3}+3 \mu_{x} \operatorname{Var}(x)+3 r d(x)\right]}{\left(E_{t}\left[V_{\tau}\right] \tau+\lambda \tau\left[\mu_{x}^{2}+\operatorname{Var}(x)\right]\right)^{3 / 2}},
\end{aligned}
$$




$$
\begin{aligned}
\text { Kurtosis }= & \frac{E_{t}\left[R_{\tau}-E_{t}\left(R_{\tau}\right)\right]^{4}}{\left[E_{t}\left[R_{\tau}-E_{t}\left(R_{\tau}\right)\right]^{2}\right]^{2}}-3 \\
= & \left(\left(3\left(\lambda \tau\left[\mu_{x}^{2}+\operatorname{Var}(x)\right]+\tau E_{t}\left[V_{\tau}\right]\right)^{2}\right.\right. \\
& +\lambda \tau\left[\mu_{x}^{4}+6 \mu_{x}^{2} \operatorname{Var}(x)\right. \\
& \left.\left.+4 \mu_{x} 3 r d(x)+4 t h(x)\right]\right) \\
& \left.\times\left(\left(E_{t}\left[V_{\tau}\right] \tau+\lambda \tau\left[\mu_{x}^{2}+\operatorname{Var}(x)\right]\right)^{2}\right)^{-1}\right)
\end{aligned}
$$$$
-3 .
$$

And the skewness and kurtosis in risk-neutral measure are given by

$$
\begin{aligned}
\overline{\text { Skewness }=} & \frac{E_{t}^{\mathrm{Q}}\left[R_{\tau}-E_{t}^{\mathrm{Q}}\left(R_{\tau}\right)\right]^{3}}{\left[E_{t}^{\mathrm{Q}}\left[R_{\tau}-E_{t}^{\mathrm{Q}}\left(R_{\tau}\right)\right]^{2}\right]^{3 / 2}} \\
= & \frac{\lambda^{\mathrm{Q}} \tau\left[\left(\mu_{x}^{\mathrm{Q}}\right)^{3}+3 \mu_{x}^{\mathrm{Q}} \operatorname{Var}^{\mathrm{Q}}(x)+3 r d^{\mathrm{Q}}(x)\right]}{\left(\tau E_{t}^{\mathrm{Q}}\left(V_{\tau}\right)+\lambda^{\mathrm{Q}} \tau\left[\left(\mu_{x}^{\mathrm{Q}}\right)^{2}+\operatorname{Var}^{\mathrm{Q}}(x)\right]\right)^{(3 / 2)},} \\
\overline{\text { Kurtosis }=} & \frac{E_{t}^{\mathrm{Q}}\left[R_{\tau}-E_{t}^{\mathrm{Q}}\left(R_{\tau}\right)\right]^{4}}{\left[E_{t}^{\mathrm{Q}}\left[R_{\tau}-E_{t}^{\mathrm{Q}}\left(R_{\tau}\right)\right]^{2}\right]^{2}-3} \\
= & \left(\left(3\left(\lambda \tau\left[\left(\mu_{x}^{\mathrm{Q}}\right)^{2}+\operatorname{Var}^{\mathrm{Q}}(x)\right]+\tau E_{t}^{\mathrm{Q}}\left[V_{\tau}\right]\right)^{2}\right.\right. \\
& +\lambda^{\mathrm{Q}} \tau\left[\left(\mu_{x}^{\mathrm{Q}}\right)^{4}+6\left(\mu_{x}^{\mathrm{Q}}\right)^{2} \operatorname{Var}^{\mathrm{Q}}(x)\right. \\
& \left.\left.\quad+4 \mu_{x}^{\mathrm{Q}} 3 r d^{\mathrm{Q}}(x)+4 t h^{\mathrm{Q}}(x)\right]\right) \\
& \left.\times\left(\left(\tau E_{t}^{\mathrm{Q}}\left(V_{\tau}\right)+\lambda^{\mathrm{Q}} \tau\left[\left(\mu_{x}^{\mathrm{Q}}\right)^{2}+\operatorname{Var}^{\mathrm{Q}}(x)\right]\right)^{2}\right)^{-1}\right)
\end{aligned}
$$$$
-3 .
$$

Corollary 15. For jump size $x$, the first moment and the second, third, and fourth central moments in the physical measure are given by

$$
\begin{gathered}
E_{t}\left(R_{\tau}\right)=\mu-\frac{1}{2} E_{t}\left(V_{\tau}\right)-\lambda E\left[\left(e^{x}-1-x\right)\right], \\
E_{t}\left[R_{\tau}-E_{t}\left(R_{\tau}\right)\right]^{2}=E_{t}\left(V_{\tau}\right) \tau+\lambda \tau E\left[x^{2}\right], \\
E_{t}\left[R_{\tau}-E_{t}\left(R_{\tau}\right)\right]^{3}=\lambda \tau E\left[x^{3}\right], \\
E_{t}\left[R_{\tau}-E_{t}\left(R_{\tau}\right)\right]^{4}=\lambda \tau E\left[x^{4}\right]+3\left(\lambda \tau E\left[x^{2}\right]+\tau E_{t}\left[V_{\tau}\right]\right)^{2} .
\end{gathered}
$$

The first moment and the second, third, and fourth central moments in the risk-neutral are given by

$$
\begin{gathered}
E_{t}^{\mathrm{Q}}\left(R_{\tau}\right)=\mu-\frac{1}{2} E_{t}^{\mathrm{Q}}\left(V_{\tau}\right)-\lambda E\left[e^{-\gamma x}\left(e^{x}-1-x\right)\right], \\
E_{t}^{\mathrm{Q}}\left[R_{\tau}-E_{t}^{\mathrm{Q}}\left(R_{\tau}\right)\right]^{2}=\tau E_{t}^{\mathrm{Q}}\left(V_{\tau}\right)+\lambda \tau E\left[e^{-\gamma x} x^{2}\right], \\
E_{t}^{\mathrm{Q}}\left[R_{\tau}-E_{t}^{\mathrm{Q}}\left(R_{\tau}\right)\right]^{3}=\lambda \tau E\left[e^{-\gamma x} x^{3}\right], \\
E_{t}^{\mathrm{Q}}\left[R_{\tau}-E_{t}^{\mathrm{Q}}\left(R_{\tau}\right)\right]^{4} \\
=\lambda \tau E\left[e^{-\gamma x} x^{4}\right]+3\left(\lambda \tau E\left[e^{-\gamma x} x^{2}\right]+\tau E_{t}^{\mathrm{Q}}\left[V_{\tau}\right]\right)^{2},
\end{gathered}
$$

where $E_{t}\left(V_{\tau}\right)=\theta-e^{-\kappa \tau}\left(\theta-V_{0}\right)$,

$$
\begin{aligned}
E_{t}^{Q}\left(V_{\tau}\right)= & \frac{\kappa \theta}{\kappa+(\gamma-\varepsilon \rho A) \varepsilon \rho} \\
& -e^{-[\kappa+(\gamma-\varepsilon \rho A) \varepsilon \rho] \tau}\left(\frac{\kappa \theta}{\kappa+(\gamma-\varepsilon \rho A) \varepsilon \rho}-V_{0}\right) .
\end{aligned}
$$

Proof. To compute central moments in the physical measure, we have $E_{t}\left(V_{\tau}\right)=\theta-e^{-\kappa \tau}\left(\theta-V_{0}\right), \mu_{x}=E(x), \operatorname{Var}(x)=$ $E\left[\left(x-\mu_{x}\right)^{2}\right]=E\left[x^{2}\right]-\mu_{x}^{2}, \operatorname{3rd}(x)=E\left[x^{3}\right]-3 \mu_{x}^{2} E\left[x^{2}\right]+2 \mu_{x}^{3}$, and $4 \operatorname{th}(x)=E\left[\left(x-\mu_{x}\right)^{4}\right]=E\left[x^{4}\right]-4 \mu_{x} E\left[x^{3}\right]+6 \mu_{x}^{2} E\left[x^{2}\right]-$ $3 \mu_{x}^{4}$.

Then, from Proposition 12, we have

$$
\begin{gathered}
E_{t}\left(R_{\tau}\right)=\mu-\frac{1}{2} E_{t}\left(V_{\tau}\right)-\lambda E\left[\left(e^{x}-1-x\right)\right], \\
E_{t}\left[R_{\tau}-E_{t}\left(R_{\tau}\right)\right]^{2}=E_{t}\left(V_{\tau}\right) \tau+\lambda \tau E\left[x^{2}\right], \\
E_{t}\left[R_{\tau}-E_{t}\left(R_{\tau}\right)\right]^{3}=\lambda \tau E\left[x^{3}\right], \\
E_{t}\left[R_{\tau}-E_{t}\left(R_{\tau}\right)\right]^{4}=\lambda \tau E\left[x^{4}\right]+3\left(\lambda \tau E\left[x^{2}\right]+\tau E_{t}\left[V_{\tau}\right]\right)^{2} .
\end{gathered}
$$

In order to compute central moments in the physical measure, from Proposition 8, we have

$$
\begin{aligned}
& E_{t}^{Q}\left(V_{\tau}\right)= \frac{\kappa \theta}{\kappa+(\gamma-\varepsilon \rho A) \varepsilon \rho} \\
&-e^{-[\kappa+(\gamma-\varepsilon \rho A) \varepsilon \rho] \tau}\left(\frac{\kappa \theta}{\kappa+(\gamma-\varepsilon \rho A) \varepsilon \rho}-V_{0}\right), \\
& \mu_{x}^{Q}=E^{Q}(x)=\frac{E\left[e^{-\gamma x} x\right]}{E\left(e^{-\gamma x}\right)}
\end{aligned}
$$




$$
\begin{gathered}
\operatorname{Var}^{\mathrm{Q}}(x)=\frac{E\left[e^{-\gamma x} x^{2}\right]}{E\left(e^{-\gamma x}\right)}-\left(\frac{E\left[e^{-\gamma x} x\right]}{E\left(e^{-\gamma x}\right)}\right)^{2} \\
3 \operatorname{rd}^{\mathrm{Q}}(x)=\frac{E\left[e^{-\gamma x} x^{3}\right]}{E\left(e^{-\gamma x}\right)}-3 \frac{E\left[e^{-\gamma x} x\right]}{E\left(e^{-\gamma x}\right)} \frac{E\left[e^{-\gamma x} x^{2}\right]}{E\left(e^{-\gamma x}\right)} \\
+2\left(\frac{E\left[e^{-\gamma x} x\right]}{E\left(e^{-\gamma x}\right)}\right)^{3}, \\
4 \operatorname{th}^{\mathrm{Q}}(x)=\frac{E\left[e^{-\gamma x} x^{4}\right]}{E\left(e^{-\gamma x}\right)}-4 \frac{E\left[e^{-\gamma x} x^{3}\right]}{E\left(e^{-\gamma x}\right)} \frac{E\left[e^{-\gamma x} x\right]}{E\left(e^{-\gamma x}\right)} \\
+6 \frac{E\left[e^{-\gamma x} x^{2}\right]}{E\left(e^{-\gamma x}\right)}\left(\frac{E\left[e^{-\gamma x} x\right]}{E\left(e^{-\gamma x}\right)}\right)^{2}-3\left(\frac{E\left[e^{-\gamma x} x\right]}{E\left(e^{-\gamma x}\right)}\right)^{4} .
\end{gathered}
$$

Then, from Proposition 12, we have

$$
\begin{gathered}
E_{t}^{\mathrm{Q}}\left(R_{\tau}\right)=\mu-\frac{1}{2} E_{t}^{\mathrm{Q}}\left(V_{\tau}\right)-\lambda E\left[e^{-\gamma x}\left(e^{x}-1-x\right)\right], \\
E_{t}^{\mathrm{Q}}\left[R_{\tau}-E_{t}^{\mathrm{Q}}\left(R_{\tau}\right)\right]^{2}=E_{t}^{\mathrm{Q}}\left(V_{\tau}\right) \tau+\lambda \tau E\left[e^{-\gamma x} x^{2}\right], \\
E_{t}^{\mathrm{Q}}\left[R_{\tau}-E_{t}^{\mathrm{Q}}\left(R_{\tau}\right)\right]^{3}=\lambda \tau E\left[e^{-\gamma x} x^{3}\right], \\
E_{t}^{\mathrm{Q}}\left[R_{\tau}-E_{t}^{\mathrm{Q}}\left(R_{\tau}\right)\right]^{4} \\
=\lambda \tau E\left[e^{-\gamma x} x^{4}\right]+3\left(\lambda \tau E\left[e^{-\gamma x} x^{2}\right]+\tau E_{t}^{\mathrm{Q}}\left[V_{\tau}\right]\right)^{2} .
\end{gathered}
$$

Corollary 16. For small risk aversion coefficient, one has the following relationship between the third central moments in the neutral-risk measure and in the physical measure:

$$
\begin{aligned}
E_{t}^{\mathrm{Q}}\left[R_{\tau}-E_{t}^{\mathrm{Q}}\left(R_{\tau}\right)\right]^{3} \approx & E_{t}\left[R_{\tau}-E_{t}\left(R_{\tau}\right)\right]^{3} \\
& -\gamma\left[E_{t}\left[R_{\tau}-E_{t}\left(R_{\tau}\right)\right]^{4}\right. \\
& \left.\quad-3\left(E_{t}\left[R_{\tau}-E_{t}\left(R_{\tau}\right)\right]^{2}\right)^{2}\right] .
\end{aligned}
$$

Proof. From Corollary 15, for small risk aversion coefficient $x$, we have

$$
\begin{aligned}
E_{t}^{Q}\left[R_{\tau}-E_{t}^{Q}\left(R_{\tau}\right)\right]^{3}= & \lambda \tau E\left[e^{-\gamma x} x^{3}\right] \\
\approx & \lambda \tau E\left[(1-\gamma x) x^{3}\right] \\
= & \lambda \tau E\left[x^{3}\right]-\gamma \lambda \tau E\left[x^{4}\right] \\
= & E_{t}\left[R_{\tau}-E_{t}\left(R_{\tau}\right)\right]^{3} \\
& -\gamma\left[E_{t}\left[R_{\tau}-E_{t}\left(R_{\tau}\right)\right]^{4}\right. \\
& \left.\quad-3\left(E_{t}\left[R_{\tau}-E_{t}\left(R_{\tau}\right)\right]^{2}\right)^{2}\right] .
\end{aligned}
$$

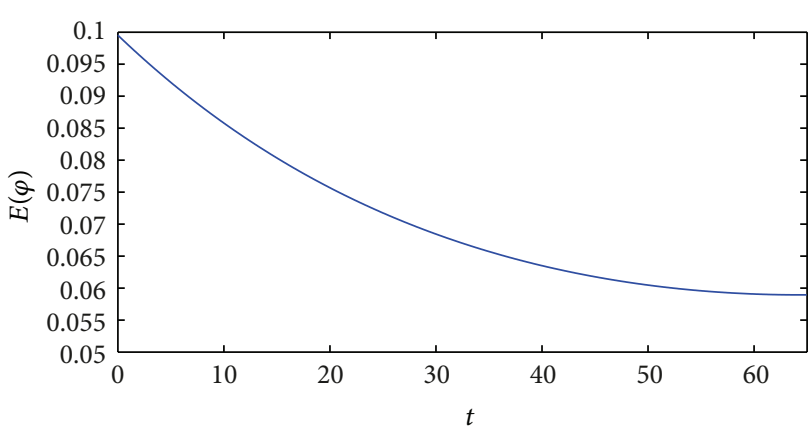

Figure 1: The expectation of $\phi$.

\section{Numerical Results}

In this section, we use our model to solve the equity premium puzzle. First, we get the long equilibrium risk premium $\bar{\phi}$ for $t \rightarrow T$, where $T$ is sufficiently large. In this case, $V_{t} \rightarrow \theta$; $A(t) \rightarrow 0$; then

$$
\bar{\phi}=\mu-r=\theta \gamma+\lambda\left(1-e^{-\gamma x}\right)\left(e^{x}-1\right) .
$$

To compare with the results from the model in [6], we take nonrandom constant jump size $x=-0.08$ and $r=0.05$, $\lambda=0.48, \mu=0.11, \theta=0.16^{2}$. Then, we obtain the relative risk aversion coefficient $\gamma=2.13$ which is the same as the numerical value in [6]. However, the risk premium $\phi$ is stochastic. We get the expectation of $\phi$ as follows:

$$
\begin{aligned}
E[\phi]= & {\left[e^{-\kappa t} V_{0}+\theta\left(1-e^{-\kappa t}\right)\right] } \\
& \times[\gamma-\varepsilon \rho A(t)]+\lambda\left(1-e^{-\gamma x}\right)\left(e^{x}-1\right) .
\end{aligned}
$$

If we assume that other parameter values in our model are $k=0.03, V_{0}=0.1, \varepsilon=0.1$, and $\rho=-0.25$, we will find that the expectation of $\phi$ tends to 0.6 from Figure 1, which is consistent with above analysis.

As we know that the risk premium $\phi$ is stochastic and it has the same stochastic characteristic with volatility $V_{t}$, the risk premium $\phi$ can be generated by a discrete scheme of system as follows:

$$
\begin{aligned}
V(t+\Delta t)= & V(t)+\kappa(\theta-V(t)) \Delta t \\
& +\varepsilon \sqrt{V(t)} \rho \omega \sqrt{\Delta t}, \quad V_{0}>0,
\end{aligned}
$$

where $\Delta t$ is the time interval and $\omega$ is a sample from the standard normal distribution.

One path of $\phi$ is given in Figure 2.

In realistic world, the risk premium is stochastic. For example, Figure 1a and Figure 5 in Graham and Harvey (2012) show that risk premium follows mean-reverting stochastic process in [41]. Thus, our model is more realistic than that in [6].

\section{Conclusions}

We successfully extend the model in [6] and obtain more general results. First, the equilibrium equity premium in 


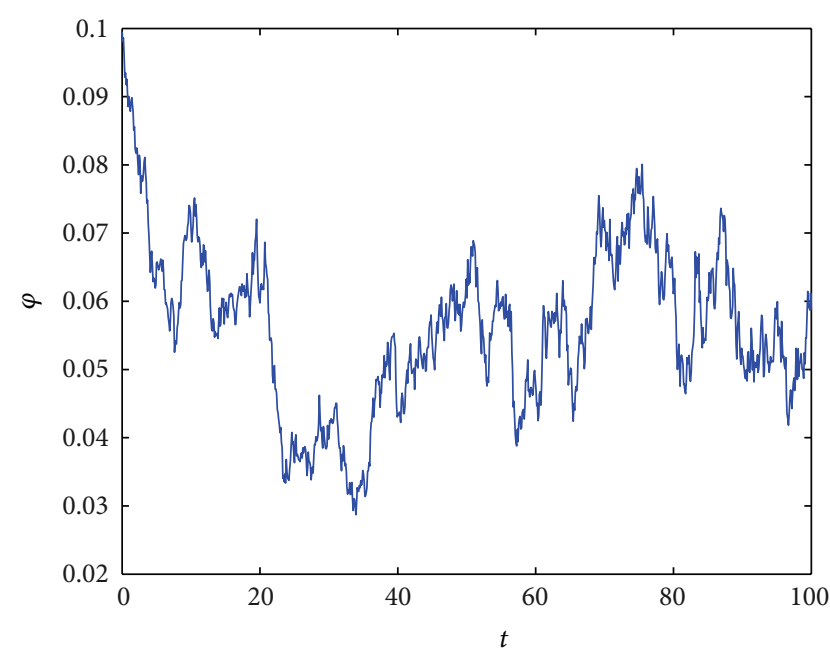

FIGURE 2: One path of $\phi$.

general equilibrium framework links not only the jump risk but also the stochastic volatility risk. Moreover, the pricing kernel with stochastic volatility is shown in this paper. We employ the Fourier transformation method to obtain the exact expression of option price. Next, we get the relationship of central moments between the physical measure and riskneutral measure. It is a vitally crucial problem in financial area. Our numerical results show that our model is more realistic than the one in [6].

\section{Acknowledgment}

This work is supported by the Fundamental Research Funds for the Central Universities (JBK130401).

\section{References}

[1] F. Black and M. Scholes, "The pricing of options and corporate liabilities," Journal of Political Economy, vol. 81, no. 3, pp. 637$659,1973$.

[2] R. C. Merton, "Option pricing when underlying stock returns are discontinuous," Journal of Financial Economics, vol. 3, no. 1-2, pp. 125-144, 1976.

[3] G. Bakshi, C. Charles, and Z. Chen, "Empirical performance of alternative option pricing models," Journal of Finance, vol. 52, no. 5, pp. 2003-2049, 1997.

[4] R. Cont and P. Tankov, Financial Modelling with Jump Processes, Chapman \& Hall, Boca Raton, Fla, USA, 2004.

[5] S. G. Kou, "A jump-diffusion model for option pricing," Management Science, vol. 48, no. 8, pp. 1086-1101, 2002.

[6] J. E. Zhang, H. Zhao, and E. C. Chang, "Equilibrium asset and option pricing under jump diffusion," Mathematical Finance, vol. 22, no. 3, pp. 538-568, 2012.

[7] P. Santa-Clara and S. C. Yan, "Volatility, and the equity premium: lessons from S\&P 500 options," The Review of Economics and Statistics, vol. 92, no. 2, pp. 435-451, 2010.

[8] A. L. Lewis, Option Valuation under Stochastic Volatility, Finance Press, Newport Beach, Calif, USA, 2000.
[9] A. Sepp, "Fourier transform for option pricing under affine jump-diffusions: an overview," In press.

[10] D. Duffie, J. Pan, and K. Singleton, "Transform analysis and asset pricing for affine jump-diffusions," Econometrica, vol. 68, no. 6, pp. 1343-1376, 2000.

[11] G. R. Duffee, "Term premia and interest rate forecasts in affine models," Journal of Finance, vol. 57, no. 1, pp. 405-443, 2002.

[12] P. Santa-Clara and S. Yan, "Jump and volatility risk and risk premia: a new model and lessons from S\&P 500 options," NBER Working Paper w10912, National Bureau of Economic Research, USA, 2004.

[13] G. Bakshi, N. Kapadia, and D. Madan, "Stock return characteristics, skew laws, and the differential pricing of individual equity options," Review of Financial Studies, vol. 16, no. 1, pp. 101-143, 2003.

[14] P. Carr and L. Wu, "Variance risk premiums," Review of Financial Studies, vol. 22, no. 3, pp. 1311-1341, 2009.

[15] A. Neuberger, "Realized skewness," Review of Financial Studies, vol. 25, no. 11, pp. 3423-3455, 2012.

[16] H. Föllmer, "Hedging of contingent claims under incomplete information," in Applied Stochastic Analysis, M. Davis and R. Elliott, Eds., vol. 5 of Stochastics Monographs, pp. 389-414, Gordon and Breach, New York, NY, USA, 1991.

[17] X. Ruan, W. Zhu, S. Li, and J. Huang, "Option pricing under risk-minimization criterion in an incomplete market with the finite difference method," Mathematical Problems in Engineering, vol. 2013, Article ID 165727, 9 pages, 2013.

[18] S. I. Boyarchenko and S. Z. Levendorskiǔ, "Option pricing for truncated Lévy processes," International Journal of Theoretical and Applied Finance, vol. 3, no. 3, pp. 549-552, 2000.

[19] M. Montero, "Renewal equations for option pricing," European Physical Journal B, vol. 65, no. 2, pp. 295-306, 2008.

[20] P. Carr, H. Geman, D. B. Madan, and M. Yor, "Pricing options on realized variance," Finance and Stochastics, vol. 9, no. 4, pp. 453-475, 2005.

[21] M. Lorig and O. Lozano-Carbasse, "Exponential Lévy-type models with stochastic volatility and stochastic jump-intensity," http://arxiv.org/abs/1205.2398 .

[22] Y. Miyahara, "Canonical martingale measures of incomplete assets markets," in Proceedings of the Seventh Japan Russia Symposium, Probability Theory and Mathematical Statistics, pp. 343352, Tokyo, Japan, 1995.

[23] Y. Miyahara, "Minimal entropy martingale measures of jump type price processes in incomplete assets markets," Asia-Pacific Financial Markets, vol. 6, no. 2, pp. 97-113, 1999.

[24] T. Chan, "Pricing contingent claims on stocks diven by Lévy processes," Annals of Applied Probability, vol. 9, no. 2, pp. 504$528,1999$.

[25] H. U. Gerber and E. S. W. Shiu, "Actuarial bridges to dynamic hedging and option pricing," Insurance, vol. 18, no. 3, pp. 183218, 1996.

[26] S. Müller, Arbitrage Pricing of Contingent Claims, Lecture Notes in Economics and Mathematical Systems, Springer, Berlin, Germany, 1985.

[27] M. Mania and R. Tevzadze, "A semimartingale backward equation and the variance-optimal martingale measure under general information flow," SIAM Journal on Control and Optimization, vol. 42, no. 5, pp. 1703-1726, 2003.

[28] O. Bobrovnytska and M. Schweizer, "Mean-variance hedging and stochastic control: beyond the Brownian setting," IEEE, 
Transactions on Automatic Control, vol. 49, no. 3, pp. 396-408, 2004.

[29] S. Hodges and A. Neuberger, "Optimal replication of contingent claims under transactions costs," Review of Futures Markets, vol. 8, no. 2, pp. 222-239, 1989.

[30] I. Karatzas, J. P. Lehoczky, S. E. Shreve, and G. L. Xu, "Martingale and duality methods for utility maximization in an incomplete market," SIAM Journal on Control and Optimization, vol. 29, no. 3, pp. 702-730, 1991.

[31] J. Cvitanić, W. Schachermayer, and H. Wang, "Utility maximization in incomplete markets with random endowment," Finance and Stochastics, vol. 5, no. 2, pp. 259-272, 2001.

[32] R. E. Lucas, Jr., "Asset prices in an exchange economy," Econometrica, vol. 46, no. 6, pp. 1429-1445, 1978.

[33] J. C. Cox, J. E. Ingersoll, Jr., and S. A. Ross, "An intertemporal general equilibrium model of asset prices," Econometrica, vol. 53, no. 2, pp. 363-384, 1985.

[34] N. Vasanttilak and M. Lee, "General equilibrium pricing of options on the market portfolio with discontinuous return," Review of Financial Studies, vol. 3, no. 4, pp. 493-521, 1990.

[35] J. Pan, "The jump-risk premia implicit in options: evidence from an integrated time-series study," Journal of Financial Economics, vol. 63, no. 1, pp. 3-50, 2002.

[36] J. Liu and J. Pan, "Dynamic derivative strategies," Journal of Financial Economics, vol. 69, no. 3, pp. 401-430, 2003.

[37] J. Liu, J. Pan, and T. Wang, "An equilibrium model of rare-event premia and its implication for option smirks," Review of Financial Studies, vol. 18, no. 1, pp. 131-164, 2005.

[38] D. S. Bates, “The market for crash risk," Journal of Economic Dynamics \& Control, vol. 32, no. 7, pp. 2291-2321, 2008.

[39] G. Chacko and S. Das, "Pricing interest rate derivatives: a general approach," Review of Financial Studies, vol. 15, no. 1, pp. 195$241,2002$.

[40] S. L. Heston, "A closed-form solution for options with stochastic volatility with applications to bond and currency options," Review of Financial Studies, vol. 6, no. 2, pp. 327-343, 1993.

[41] J. Graham and C. Harvey, "The equity risk premium in 2012," Social Science Research Network, 2012. 


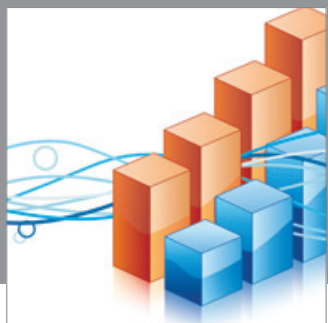

Advances in

Operations Research

mansans

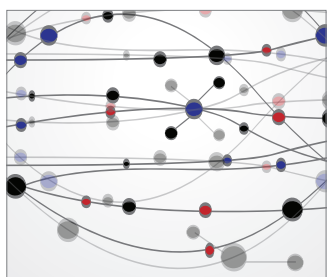

The Scientific World Journal
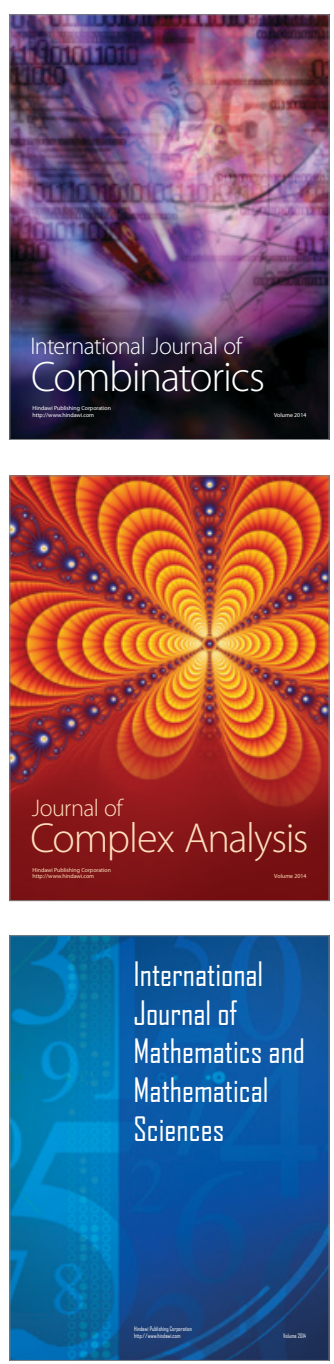
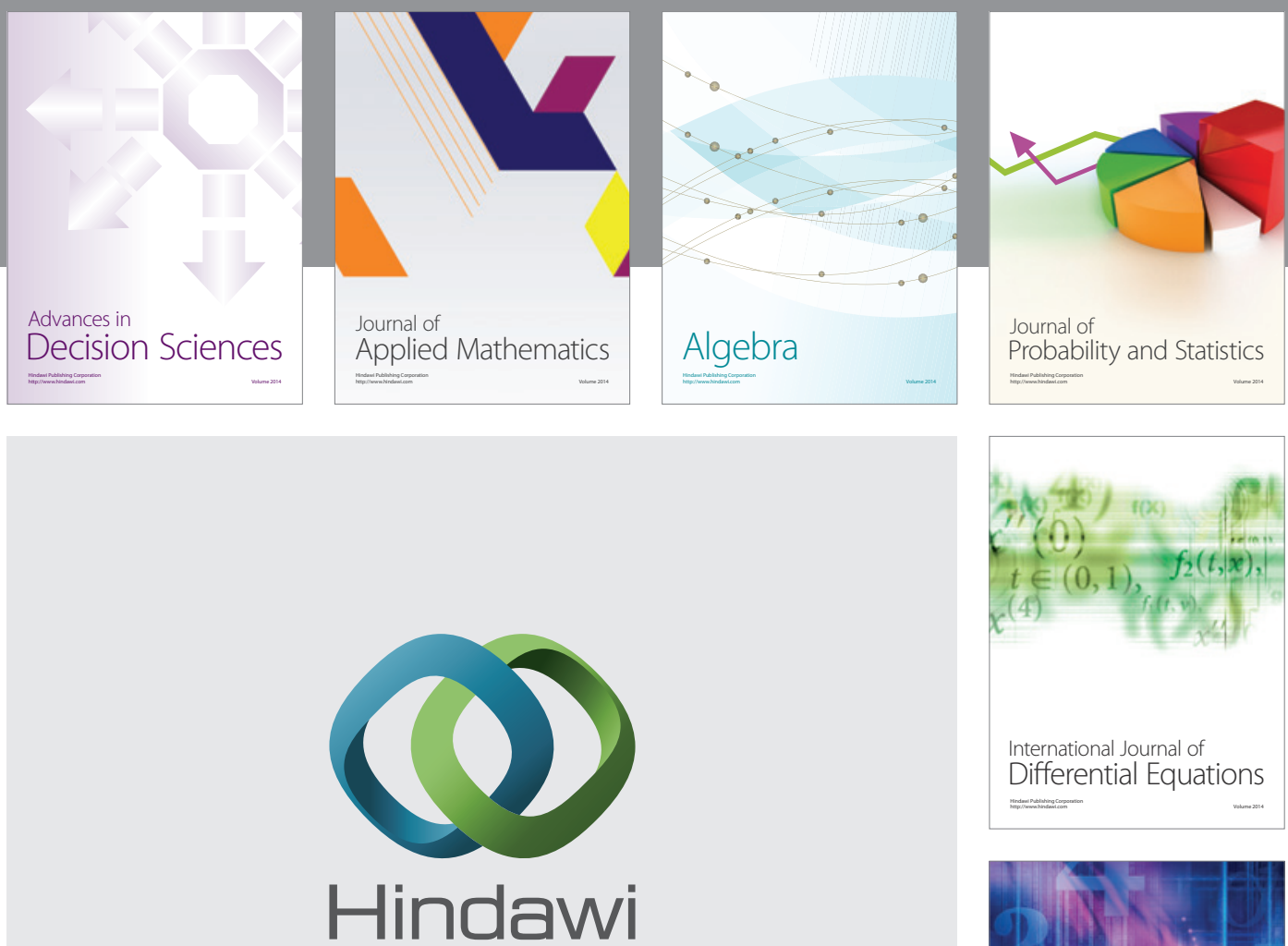

Submit your manuscripts at http://www.hindawi.com
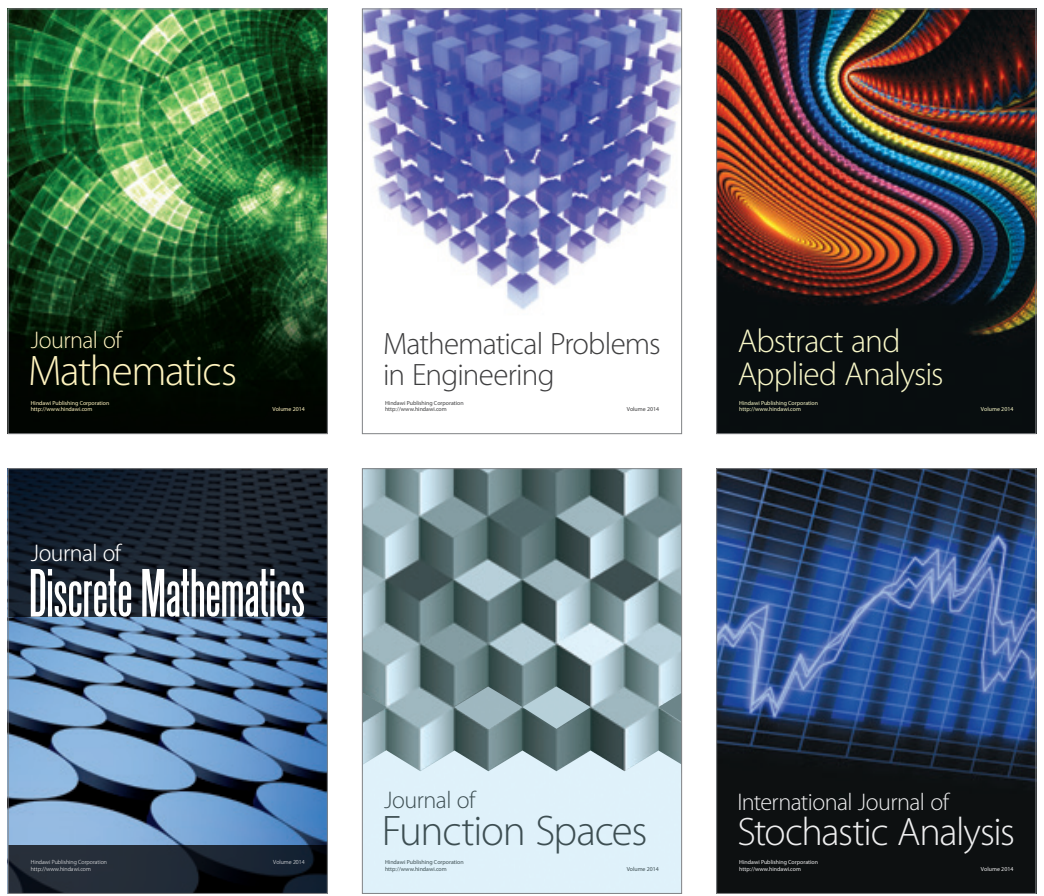

Journal of

Function Spaces

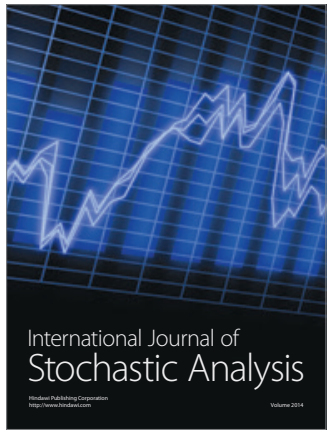

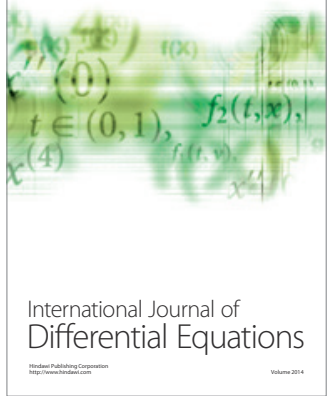
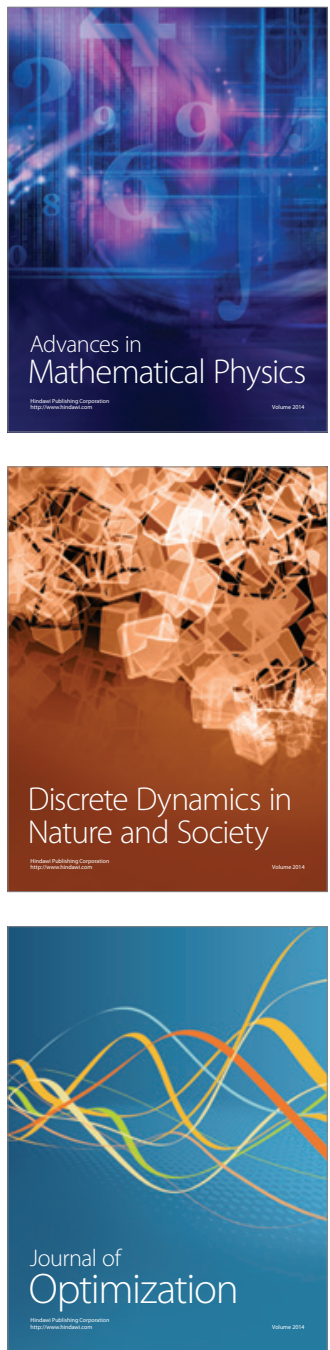